Memorias del VII Encuentro Nacional de Experiencias en la Enseñanza de la Biología y la Educación Ambiental y II Congreso Nacional de Investigación en la Enseñanza de la Biología

\title{
HACIA UNA ESCUELA PARA LA SOCIEDAD: UNA PERSPECTIVA AMBIENTAL DESDE LA ECONOMÍA AZUL
}

\section{TOWARD A SCHOOL FOR THE SOCIETY: AN ENVIRONMENTAL PERSPECTIVE FROM THE BLUE ECONOMY}

\section{Mónica García ${ }^{1}$ \\ Dino Segura ${ }^{2}$}

Resumen (178/250):

Incluir la ética ambiental como una disciplina en el currículo al interior de las instituciones educativas no asegura un proceso significativo de formación en el campo ambiental; pues para esto, se requiere de una transformación profunda del sistema educativo que valore no solo la necesidad de pensar en los problemas del contexto sino de formar ciudadanos que cuestionen los procesos de homogenización cultural y biológica actualmente imperantes.

La propuesta de la Economía Azul de Gunter Pauli, presenta una perspectiva que posibilita contrapesar los procesos de globalización, haciendo uso de los conocimientos que se tienen sobre la forma en como la naturaleza soluciona sus problemas para sostenerse y aprovechar de la mejor forma los productos e insumos que resultan de cada proceso.

El presente artículo comparte las reflexiones y describe la experiencia de la Escuela Pedagógica Experimental, desde el momento que retoma los planteamientos de la Economía Azul para enriquecer su propuesta y consolidar nueve proyectos ambientales (mediante la estrategia pedagógica ATAs), los cuales han resultado del estudio de problemas genuinos presentes en la cotidianidad de la comunidad escolar.

\section{Abstract (250):}

Including the environmental ethics as a subject in the curriculum of the educational institutions does not guarantee a meaningful process of preparation

\footnotetext{
${ }^{1}$ Magister en Educación de la Universidad Pedagógica Nacional. Investigadora Corporación Escuela Pedagógica Experimental. Profesora de Ciencias y Educación Ambiental. monica.garcia@epe.edu.co

${ }^{2}$ Magíster en Fisica de la Universidad de Leipzig (Alemania) y Magíster en Educación. States University of New York. Coodinador General de la Corporación Escuela Pedagógica Experimental. dino.segura@epe.edu.co
} 
Bio-grafía Escritos sobre la Biología y su Enseñanza.

Edición Extra-Ordinaria. ISSN 2027-1034 P. p49-57

Memorias del VII Encuentro Nacional de Experiencias en la Enseñanza de la

Biología y la Educación Ambiental y II Congreso Nacional de Investigación en la Enseñanza de la Biología

in environmental issues. In order to achieve this, it may be necessary a deep transformation of the educational system, considering the problems of the context and educating citizens who are able to inquire about the biological and cultural homogenization process that is prevailing in the actuality.

The concept of The Blue Economy proposed by Gunter Pauli can be define as an option of balance the globalization processes through the implementation of the nature behavior. This means, to take into account the way in which nature solves its problems, remaining sustainable and taking advantage of all the products that result from each process.

This article deals with the findings, thoughts and experiences that the Escuela Pedagógica Experimental has had from the moment it approached to the Blue Economy model; adopting its proposal and (through the ATAs teaching strategy) consolidating nine environmental projects that resulted as a consequence from genuine issues present in the everyday circumstances the scholar community has to face.

Palabras Clave: Ética Ambiental, Economía Azul, Actividad Totalidad Abiertas, Trabajo por Proyectos.

Keywords: Environmental Ethics, Blue Economy, Actividades Totalidad Abiertas, Working with Projects.

\section{Introducción}

Aunque desde mediados de los años 70 se empezó a ver la necesidad de abordar en las universidades la ética ambiental como una disciplina debido a la urgencia por analizar críticamente y transformar las relaciones que hasta el momento habían construido las sociedades humanas con el ambiente del que forman parte (Rozzi, et al., 2001); para muchas comunidades indígenas y culturas ancestrales, la ética ha sido un tema histórico y fundamental en sus formas de organización social, en busca de una relación más respetuosa con el mundo natural. Es por ello que se justifica la necesidad de construir espacios de diálogo en donde no solo sean reconocidos los conocimientos (relativamente recientes) elaborados por la comunidad científica (antropólogos, históricos, ecólogos, físicos, filósofos, entre otros), para superar la crisis ambiental, sino también aquellos saberes que durante muchos años han aportado en la construcción de relaciones sostenibles con el ambiente. 
Memorias del VII Encuentro Nacional de Experiencias en la Enseñanza de la

Biología y la Educación Ambiental y II Congreso Nacional de Investigación en la Enseñanza de la Biología

Así, la ética ambiental además de ser un tema de moda en los currículos de las instituciones educativas impuestos por la "cultura dominante" (en palabras de Rigoberta Menchú Tum, primera mujer de raza indígena en recibir un Premio Nobel, 1992) como necesidades mundiales; es una necesidad en nuestros territorios para contrapesar los procesos de globalización y de homogenización biológica y cultural. Al respecto, es muy importante que conozcamos los saberes que han construido, por ejemplo, nuestras comunidades campesinas, basados en prácticas ambientalmente "sostenibles" ${ }^{3}$, y que presentan una alternativa económica y política diferente al modelo de mercado libre actualmente imperante.

Desde esta perspectiva, la propuesta educativa de la Escuela Pedagógica Experimental en el ámbito de lo ambiental retoma los planteamientos de Gunter Pauli (2011), en su libro sobre Economía Azul, cuando menciona la importancia de transformar la visión tradicional de la enseñanza (hasta ahora centrada en los contenidos); que ha aportado muy poco en la solución a problemas locales. De tal forma que, en concordancia con el autor, "quizá la mayor libertad que podamos ofrecer a nuestro hijos sea permitirles pensar de otra manera y, lo que es más importante, actuar de otra manera (...) si enseñamos a los niños solo lo que conocemos, nunca podrán hacerlo mejor que nosotros" (Pauli, 2011, p.17). Como plantea Ricardo Rozzi (1997) (citado por Rozzi, et al. 2001, p. 326) una ética ambiental "demanda también transformar los sistemas educacionales adoptando aproximaciones internas culturales con participación de comunidades indígenas y campesinas, trabajadores urbanos y rurales, donde los estudiantes sean educados para ser ciudadanos y no meramente consumidores".

Entonces, los proyectos educativos ambientales fundamentados en los principios de la Economía Azul, además de proponer como elemento definitivo el diálogo de saberes, tienen como intención transformar el círculo vicioso que actualmente alimenta la crisis ambiental, en donde elementos como: la explosión demográfica, el crecimiento industrial desbocado, el declive de valores éticos y la degradación medioambiental, se retroalimentan positivamente. Así, se hace necesario empezar a cuestionar los principios de la economía de mercado libre, conceptualizados por el filósofo escocés Adam Smith (quien introdujo el interés individual como un aspecto de la naturaleza humana), y que suponen equivocadamente "que los costos y beneficios del

${ }_{3}^{3}$ Entendiendo sostenibilidad como aquel desarrollo que satisface las necesidades de la generación presente sin comprometer la capacidad de las generaciones futuras para satisfacer sus propias hecesidades (Willches-Chaux, 1993), 
Memorias del VII Encuentro Nacional de Experiencias en la Enseñanza de la

Biología y la Educación Ambiental y II Congreso Nacional de Investigación en la Enseñanza de la Biología

intercambio económico afectan solo a los participantes en la transacción" (Rozzi, et al. 2001, p.262), desde una mirada de sistema cerrado.

Como resultado evidente de estas dinámicas de mercado podemos encontrar que se han promovido procesos de expropiación y concentración de la propiedad de la tierra, migraciones forzadas de las poblaciones rurales a las grandes ciudades, desplazamientos obligados de las personas a áreas ambientales protegidas, concentración de bienes y beneficios en un grupo pequeño de personas y corporaciones, entre otros. En tal sentido, se hace relevante que toda propuesta ambiental, ya sea a nivel escolar o no, plantee alternativas a la economía, ya que el actual modelo considera "las transacciones del mercado como un sistema cerrado, independiente de contextos sociales y ambientales más amplios" (Rozzi, et al. 2001, p.262), al punto que supone por ejemplo, que en el proceso de extracción del oro solo se afectan los involucrados directamente al momento de la transacción; y se omiten los costos ambientales y sociales provocados por esta actividad.

La Economía Azul, como propuesta ambiental, también plantea alternativas al modelo económico actual, cuando busca generar modos de vida sostenible y, al mismo tiempo, satisfacer las necesidades básicas de todos, sin excluir a ninguna persona; de tal forma que, las acciones ambientales no se centran, como hasta ahora ha ocurrido, en la producción de elementos que sean biodegradables y que buscan solo mitigar el impacto del hombre en el medio (a propósito de la Economía Verde), sino en construir ejes de acción, fundamentados en los conocimientos que se tienen sobre los sistemas naturales.

En otras palabras, es importante que empecemos a ver la economía como un sistema abierto en el que ocurre un intercambio permanente de materia y energía entre las diferentes organizaciones sociales y culturas con los sistemas naturales. En este caso, por ejemplo se puede pensar en imitar la eficiencia sin residuos de los ecosistemas, pues, con seguridad, como plantea Gunter Pauli (2011), muchos de los problemas de sostenibilidad a los que se enfrenta la humanidad ya los ha resuelto el mundo natural, de forma que estudiar los ecosistemas nos puede permitir aprender otras formas de ver los sistemas de producción y consumo, eliminando, el concepto de desecho e imitando el ciclado de nutrientes y energía tal como sucede en la naturaleza.

Los proyectos ambientales que se construyeron en el marco de la economía azul, al interior de la Escuela Pedagógica Experimental, retoman varios de los principios anteriormente mencionados, y desde su inicio en el año 2012, 
Bio-grafía Escritos sobre La Biología y su Enseñanza.

Edición Extra-Ordinaria. ISSN 2027-1034 P. p 49-57

Memorias del VII Encuentro Nacional de Experiencias en la Enseñanza de la

Biología y la Educación Ambiental y II Congreso Nacional de Investigación en la Enseñanza de la Biología

pretenden transformar las actitudes y acciones frente al concepto de desecho, pues lo que es desecho para unos es materia disponible para otros. Realmente, el problema está en que malgastamos los residuos que generamos, y hacemos económicamente insostenible este sistema.

\section{Metodología}

Desde el año 1989 la Escuela Pedagógica Experimental, como resultado de uno de sus procesos de investigación en el ámbito pedagógico, propuso una estrategia para el trabajo en el aula: las Actividades Totalidad Abiertas (ATAs); la cual, como se ha escrito en numerosas publicaciones, no solo parte de una perspectiva diferente de concebir el sujeto frente a los procesos de construcción de conocimiento, sino de transformar las prácticas sobre el aprendizaje y de esta forma, proponer otra manera de organización en el ambiente educativo.

A manera de resumen, las ATAs proponen entre algunos elementos esenciales para su realización (Segura, et al., 1995):

a) La construcción de una tensión cognoscitiva-afectiva de deseo entre el individuo y su actividad,

b) El mantenimiento del interés por lo que se realiza en clase,

c) El compromiso con un problema genuino,

d) Las situaciones colectivas de búsqueda en las que la libertad de pensamiento, la diferencia, la posibilidad de disentir y la creatividad e imaginación aportan a la democracia,

e) La articulación de las actividades que se realizan con valores como la anticipación y el diseño.

f) El reconocimiento de diferentes fuentes de conocimiento y saberes,

g) El compromiso con el contexto, y

h) La acción frente al problema inicial.

Todos estos elementos se constituyen en una alternativa para trabajar en la escuela a partir de proyectos, y exigen cambios estructurales en la organización de la institución educativa; tanto en la forma de pensar el currículo, como en la manera como los maestros asumen el acompañamiento o asesoría pedagógica. En otras palabras, la estrategia "ATAs" metodológicamente se encuentra fundamentada en el trabajo por proyectos, y por tanto implica: 
Bio-grafía Escritos sobre La Biología y su Enseñanza.

Edición Extra-Ordinaria. ISSN 2027-1034 P. p49-57

Memorias del VII Encuentro Nacional de Experiencias en la Enseñanza de la Biología y la Educación Ambiental y II Congreso Nacional de Investigación en la Enseñanza de la Biología

> Flexibilidad en el currículo, pues cuando los proyectos son genuinos superan el horario destinado para las asignaturas.

$>$

> Maestros que quieran aprender, ya que sin duda existen maestros aferrados a la información que les proporcionan las disciplinas, y es necesario que se cambien las dinámicas. Debemos aprender lo que queremos aprender, y no se trata de estar simplemente alertas frente a lo que OTROS quieran enseñarnos.

$>$ Voluntad, para mantenerse en las búsquedas a pesar de las dificultades.

\section{Resultados y Discusión}

La Escuela Pedagógica Experimental, desde su constitución en el año 1977 ha planteado como pilar dentro de su proyecto educativo "el compromiso de la escuela con el contexto"; por lo que más allá de pensar en la constitución de un currículo ha logrado consolidar en sus dinámicas propuestas de trabajo relacionadas directamente con la formación de ciudadanos comprometidos con las problemáticas del entorno, y dispuestos a transformar sus prácticas en acciones sostenibles y amigables con el ambiente. En este sentido, con el pasar de los años proyectos como: Loncheras Saludables, Conservación de Especies Nativas, Silvicultura y Recuperación del Arboreto, y Recuperación del suelo para siembra de plantas ornamentales y medicinales, han logrado establecerse y ser reconocidos por la comunidad.

En los dos últimos años, el acercamiento de la Escuela con los planteamientos de Gunter Pauli sobre "Economía Azul", ha enriquecido las reflexiones, prácticas y proyecciones de las propuestas ambientales al interior de la institución. De forma que al inicio del año 2012, las discusiones permitieron construir en un trabajo conjunto entre estudiantes, maestros y padres de familia, dos proyectos que en su desarrollo cuestionaron algunas de las dinámicas de consumo que teníamos como comunidad; uno de estos sobre el tratamiento de residuos sólidos, y otro, sobre loncheras saludables. Estos dos proyectos nos dieron en principio la posibilidad de participar en una plenaria en el Capitolio Nacional, en donde se expuso la importancia de construir otra mirada sobre nuestras acciones cotidianas y de aunar esfuerzos para construir un mundo diferente, partiendo de la premisa de que en los detalles pequeños también se encuentran grandes cambios. 
Bio-grafía Escritos sobre La Biología y su Enseñanza.

Edición Extra-Ordinaria. ISSN 2027-1034 P. p49-57

Memorias del VII Encuentro Nacional de Experiencias en la Enseñanza de la Biología y la Educación Ambiental y II Congreso Nacional de Investigación en la Enseñanza de la Biología

n el presente año 2013, el éxito de la experiencia anterior permitió dar apertura a la creación de nueve proyectos ambientales fundamentados en la Economía Azul y en una lectura crítica sobre el contexto. Estos son: plantas ornamentales y medicinales, aprovechamiento de agua lluvia, recuperación del arboreto, lumbricultivo, tratamiento de residuos sólidos, mermeladas, tejidos y telares, construcción de juguetes y recuperación de la quebrada (ver ilustración 1).

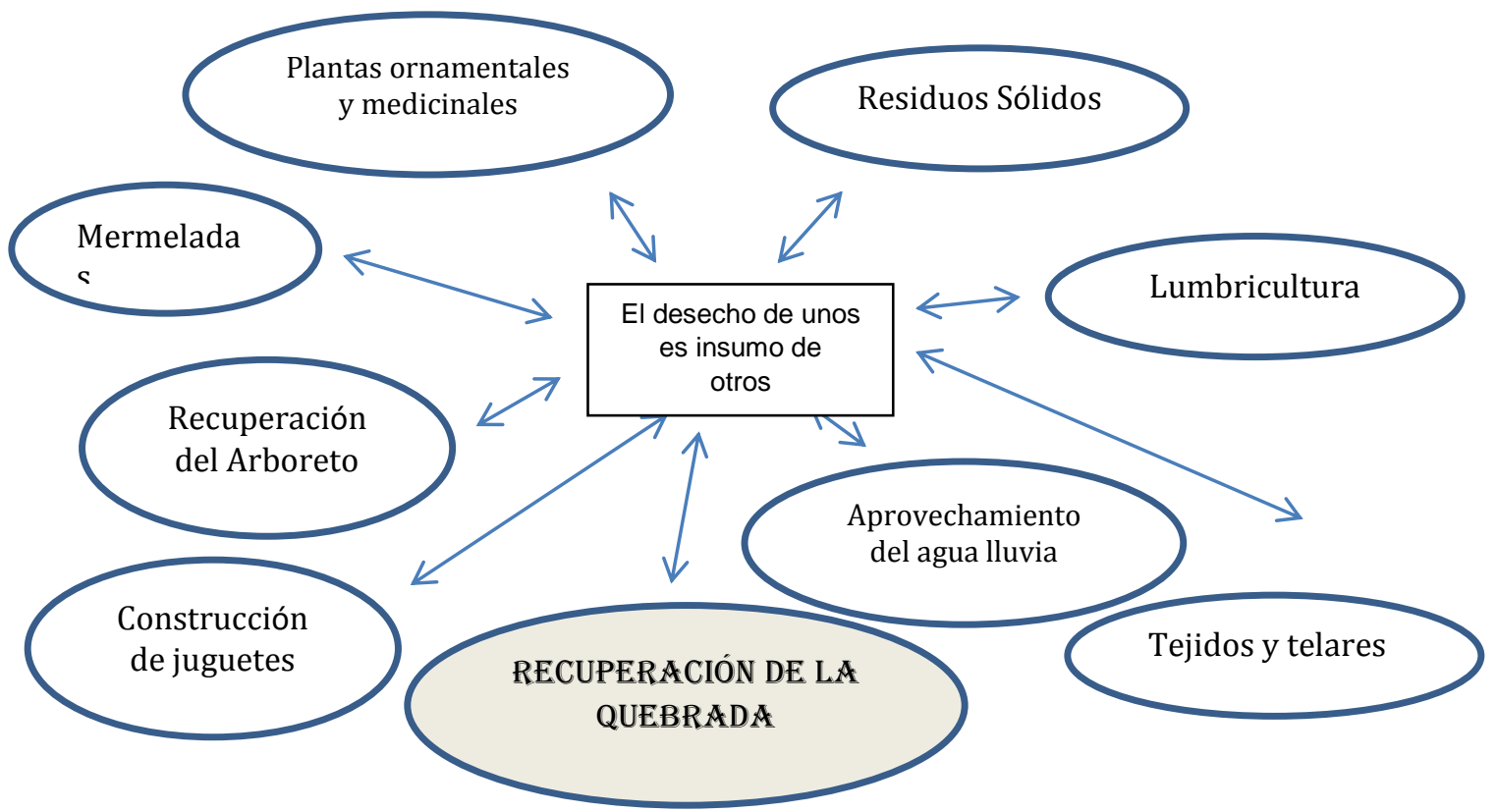

Ilustración 1. Proyectos Ambientales en el marco de la Economía Azul.

Todos ellos, dentro de su dinámica de trabajo han mantenido una búsqueda permanente por encontrar relaciones entre unos y otros, tomando como eje central la discusión sobre el principio de: el residuo de unos es insumo para otros; constituyendo éste un primer resultado. A su vez, en cada uno de los proyectos se han hecho evidentes otros resultados relevantes al momento de pensar la formación integral y significativa para los estudiantes. Estos son:

- Se ha fortalecido los vínculos con la comunidad aledaña a la Escuela (habitantes del sector "vecinos", jardines infantiles, organizaciones comunitarias "casa-taller las Moyas", IED Monteverde) para unir esfuerzos en el desarrollo de las propuestas.

- Se han construido redes de trabajo con especialistas e instituciones interesadas en apoyar cada uno de los proyectos, como: el Jardín Botánico de Bogotá, el acueducto comunitario ACUALCOS, 
Bio-grafía Escritos sobre la Biología y su Enseñanza.

Edición Extra-Ordinaria. ISSN 2027-1034 P.p 49-57

Memorias del VII Encuentro Nacional de Experiencias en la Enseñanza de la

Biología y la Educación Ambiental y II Congreso Nacional de Investigación en la Enseñanza de la Biología

Conservación Internacional y el proyecto de Recuperación de Quebradas de Chapinero.

- Se ha hecho un reconocimiento especial a la experiencia de los campesinos del sector (invitándolos también a ser especialistas en el tema) y a los saberes presentes en las culturas indígenas del país.

- Se han abordado oportunamente las informaciones provenientes de las disciplinas para profundizar en el tratamiento de los problemas desde una perspectiva significativa.

- Se ha aportado en la consolidación de una propuesta pedagógica fundamentada en el trabajo autónomo, participativo y propositivo por parte de los estudiantes.

- Se ha transformado el papel del docente en los procesos de formación.

- Se ha logrado atrapar el interés y la participación de padres y maestros (con formaciones académicas convencionalmente desvinculadas al ámbito ambiental) realmente comprometidos con las problemáticas de su entorno.

Los resultados de los proyectos se muestran con detalle en la página web de la Escuela: www.epe.edu.co; a través de videos y álbumes de fotos, documentos escritos, reportajes y diarios de campo.

\section{Conclusiones}

La biodegradabilidad y renovabilidad no necesariamente equivalen a la sostenibilidad, pues la sostenibilidad sólo es factible cuando se piensan sistemas económicos de producción y consumo alternativos, que de manera eficiente respondan a las necesidad básicas de todos (sin segregar 0 discriminar) usando los elementos que nos ofrece el entorno local.

Los principios de la economía azul pueden aportar en la construcción de proyectos ambientales al interior de las instituciones educativas, pues además de tener un impacto sobre la comunidad local en su forma de relacionarse con el ambiente en el que habitan, hace una contribución relevante a las dinámicas ambientales al transformar el concepto de desecho. 
Bio-grafía Escritos sobre La Biología y su Enseñanza.

Edición Extra-Ordinaria. ISSN 2027-1034 P. p 49-57

Memorias del VII Encuentro Nacional de Experiencias en la Enseñanza de la

Biología y la Educación Ambiental y II Congreso Nacional de Investigación en la Enseñanza de la Biología

Desde el punto de vista formativo, el trabajo en proyectos genuinos, esto es, en proyectos que verdaderamente existen y requieren de intervención, ofrece a la vida escolar elementos importantes:

$>$ Se encuentra el valor del conocimiento, lo que se aprende vale la pena.

$>$ Las dificultades se convierten en ocasión para profundizar, por ejemplo, si se requieren arbolitos, tendremos que estudiar las diferentes maneras de tratar las semillas y las diversas formas de reproducción de los árboles.

> La generación de ventajas económicas marca una perspectiva importante frente a la pobreza y la falta de puestos de trabajo.

$>$ La complejidad de las relaciones entre proyectos diferentes exige del trabajo colaborativo y de la planeación conjunta.

> Se plantea una alternativa frente a la globalización que desemboca en la educación en la neutralidad de los aprendizajes y la inutilidad de los contenidos.

\section{Bibliografía}

Pauli, G. (2011). La economía azul: 100 años, 100 innovaciones, 100 millones de empleos. México, D.F.: Tusquets Editores.

Rozzi, R., Primack, R., Feisinger, P., Dirzo, R., \& Massardo, F. (2001). Fundamentos de conservación biológica: Perspectivas latinoamericanas. México, D.F.: Fondo de Cultura Económica.

Segura, D. \& Molina, A. (1995). Vivencias de conocimiento y cambio cultural. Bogotá (Colombia): Colección Polémica Educativa, Escuela Pedagógica Expeirmental-Colciencias.

Wilches-Chaux, G. (1993). ¿Y qué es eso de desarrollo sostenible?. Colombia: Departamento Nacional de Planeación, Corpes-Presidencia de la República.

Páginas de apoyo:

Menchú, R. Interculturalidad como utopía [documento PDF]. En http://200.10.23.169/trabajados/menchu.pdf 